\title{
Phase relationships between two or more interacting processes from one-dimensional time series. II. Application to heart-rate-variability data
}

\author{
N. B. Janson, ${ }^{1}$ A. G. Balanov, ${ }^{1}$ V. S. Anishchenko, ${ }^{2}$ and P. V. E. McClintock ${ }^{1}$ \\ ${ }^{1}$ Department of Physics, Lancaster University, Lancaster, LA1 4YB, United Kingdom \\ ${ }^{2}$ Department of Physics, Saratov State University, Astrahanskaya 83, 410026, Saratov, Russia
}

(Received 27 July 2001; published 15 February 2002)

\begin{abstract}
The recently proposed approach to detect synchronization from univariate data is applied to heart-ratevariability (HRV) data from ten healthy humans. The approach involves introducing angles for return times map and studying their behavior. For filtered human HRV data, it is demonstrated that: (i) in many of the subjects studied, interactions between different processes within the cardiovascular system can be considered as weak, and the angles can be well described by the derived model; (ii) in some of the subjects the strengths of the interactions between the processes are sufficiently large that the angles map has a distinctive structure, which is not captured by our model; (iii) synchronization between the processes involved can often be detected; (iv) the instantaneous radii are rather disordered.
\end{abstract}

DOI: 10.1103/PhysRevE.65.036212

PACS number(s): 05.45.Xt, 05.45.Tp, 87.19.Hh

\section{INTRODUCTION}

A general approach has recently been proposed $[1,2]$ for the detection of phase synchronization (or its absence) between two or more interacting processes based on the analysis of univariate data. The approach consists in extracting return times from a continuous one-dimensional observable, reconstructing the return times map by delay embedding, and extracting phase angles. In the immediately preceding paper [2], hereinafter referred to as Part I, we demonstrated analytically and numerically that for two interacting processes these angles are in one-to-one correspondence with the conventional phase difference, and can thus be taken as an indication of synchronization or otherwise. The same method was also extended to the case of several processes interacting in the presence of noise, and its workability was illustrated numerically for the case of three processes.

In the present paper we apply this approach to heart-ratevariability (HRV) data from healthy human subjects in order to learn whether or not synchronization occurs for three of the most significant processes operating within the cardiovascular system, namely, the main heart rhythm, respiration, and the process whose basic frequency is close to $0.1 \mathrm{~Hz}$. The HRV data are in the form of $R-R$ intervals extracted from electrocardiogrammes (ECGs).

\section{DESCRIPTION OF DATA}

Figure 1(a) shows a typical human ECG. The so-called $R$ peaks, which are the largest ones, are indicated. The time intervals $T_{i}$ between the two successive $R$ peaks are usually called $R-R$ intervals. They represent the time intervals between the two consecutive heart beats and in terms of nonlinear dynamics the time intervals between the trajectory's return to a secant plane defined by the value of a threshold, i.e., return times. It is widely accepted that the human ECG has rather complex Fourier (and wavelet) spectra with welldistinguished characteristic peaks, three of them being especially noticeable: a schematic Fourier power spectrum for a typical ECG is shown in Fig. 1(b). Usually these peaks are associated with the rhythmic activity of certain physiological processes within cardiovascular system (CVS): $f_{\text {ahr }}$ defines the average heart rate; $f_{\text {resp }}$ is associated with respiration process; the origin of $f_{2} \sim 0.1 \mathrm{~Hz}$ is not quite clear at present-it is variously attributed to the sympathetic and parasympathetic nervous activity [3], to the baroreflex loop [4], and to the intrinsic myogenic activity of the vascular smooth muscles [5]. At least two more distinguishable spectral peaks, at frequencies of about 0.01 and $0.03 \mathrm{~Hz}$ (not shown here), have recently been recognized [5]. However, on the small observation times with which we deal in the present research, they cannot be detected with confidence and, in the framework of this paper, we will treat them as additional manifestations of nonstationarity.

Qualitatively the same Fourier spectra can be obtained [6] from $R-R$ intervals using a technique suggested in [7].

Thus, the dynamics of $R-R$ intervals result from the complex interaction of several processes with different timescales. With the exception of respiration and heart rate, there is usually (but cf. [8]) no possibility of gaining any knowledge about the phase or amplitude relationships between them with the use of noninvasive methods. It is, therefore, interesting to find out whether our technique can be helpful in order to detect the presence or absence of phase synchronization between the processes involved based solely on studies of the sequences of $R-R$ intervals.

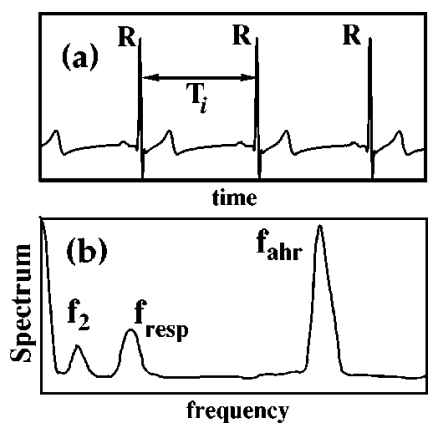

FIG. 1. (a) Typical human electrocardiogram (ECG). (b) Schematic power spectrum in decibels for a typical human ECG. 
Data were recorded from 10 healthy students and young researchers of Saratov State University. Each ECG was registered while the subject was resting in an armchair, over a period of 5-10 min, using a sampling rate of $180 \mathrm{~Hz}$. For a test example of bivariate data, where both ECG and respiration signals were required, measurements were made at Lancaster University during 3 min with a sampling rate of 400 $\mathrm{Hz}$ from a young healthy subject undergoing paced respiration with frequency of $0.5 \mathrm{~Hz}$.

\section{REMOVING THE FLOATING AVERAGE VALUE}

As it was noted in Part I (Ref. 45) the return times of a nonstationary process usually oscillate around some randomly floating average value. In terms of the angles of return times map it means that the origin of this map is floating randomly. Angles extracted from such data are usually highly disordered and the useful phase information appears to be smeared. We are interested in the oscillations of return times around the average value, thus in order to gain "clean" phase information we advocate removal of this floating origin from return times prior to the extraction of angles. Any one of several existing methods can be used for this purpose, but here we discuss just two of them. We first show how they transform purely noisy data when no interactions take place (Sec. III B), and then demonstrate their effect on a typical example of human HRV data (Sec. IV).

\section{A. Description of filtering methods}

In the present paper we use two different methods. The first of these consists of computing the analog of the second derivative of the original discrete time series $x(i)$,

$$
x_{\mathrm{der}}(i)=\frac{x(i+1)+x(i-1)-2 x(i)}{2} .
$$

Let us refer to this method as to the method of derivatives.

The second method is an extension of the well-known detrending technique. A local average is defined within a temporal window moving along the dataset which is then deduced from each datapoint. The only distinction of our method is that the size of the temporal window is not constant along the dataset. One window includes all points between two successive extrema (maximum and minimum, etc.) of a discrete signal, including the extrema themselves. After the local average is computed within each window, its value is attributed to the time in the middle of the window. All such averages are then connected by straight lines by means of linear interpolation. Finally, from each original datapoint the value of the resultant graph is deduced [see also Fig. 3(c)]. In what follows we will call this technique the method of differences.

Further, while plotting the filtered discrete data we will add them to the average value of the original unfiltered dataset.

\section{B. Noisy oscillatory process without any interaction}

Before attempting to apply our technique in practice to investigate interactions between oscillatory processes we
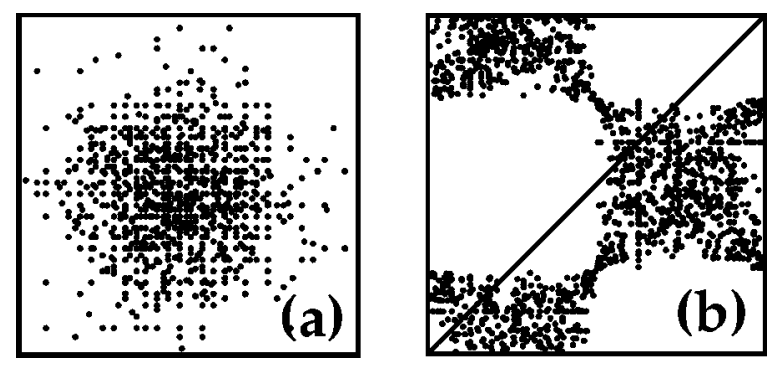

FIG. 2. Van der Pol system forced only by Gaussian white noise of intensity $D=10^{-5}$ : (a) return times map; (b) angles map.

first need to know how the map of angles of return times behaves in the case when there are no deterministic interactions between processes being close to periodic, i.e., where only one noisy periodic oscillatory process occurs. Consider the Van der Pol oscillator in the presence of noise, i.e., Eq. (2) of Part I in the absence of external forcing $(C=0)$, with noise intensity $D=0.00001, \epsilon=0.1$, and $\omega=1$. Let us record times $t_{i}$ when the phase trajectory intersects the level $x=0$ in one direction. The map for return times is presented in Fig. 2(a) while the map for angles of return times in Fig. 2(b). It is easy to be convinced that the latter map contains no structure by comparing it with those of Figs. 3 or 6 of Part I, representing Eqs. (11) or (19), respectively.

In order to check that the distinct structure that may appear in map for angles is not merely due to the filtration procedures that we have used, we now apply the above filters to these purely noisy data. The results obtained after filtration are presented in Fig. 3(a) and 3(b). The presence of some structure in the resulting angles maps is quite obvious herethough it is far from being one dimensional, and neither is there any possibility of modeling these maps by means of Eq. (19) in Part I. Note that the points concentrate in very specific regions, namely, between the return functions of Eq. (11) in Part I for $\xi=\frac{1}{3}$ and $\xi=\frac{1}{2}$ shown by gray dashed lines, and that they fill densely the interior and the vicinity of this
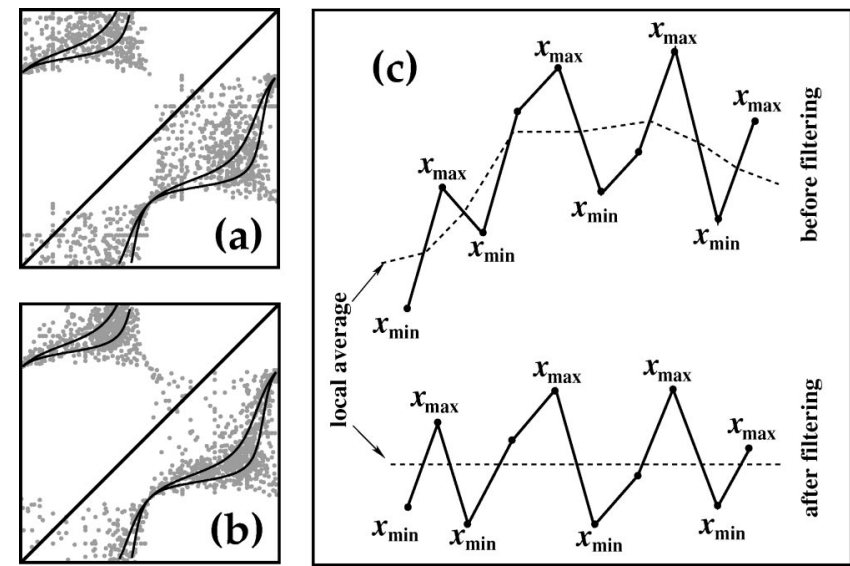

FIG. 3. Results of the application of the two filtering techniques to the purely noisy return times illustrated in Fig. 2(a). Angles maps are shown after applying differences (a) and derivatives (b) methods, respectively. Solid lines in (a) and (b) show return functions of Eq. (11) in Part I for $\xi=1 / 3$ (uppers) and $\xi=1 / 2$ (lowers). In (c) the operation of filtering by differences is illustrated for the noise data. 


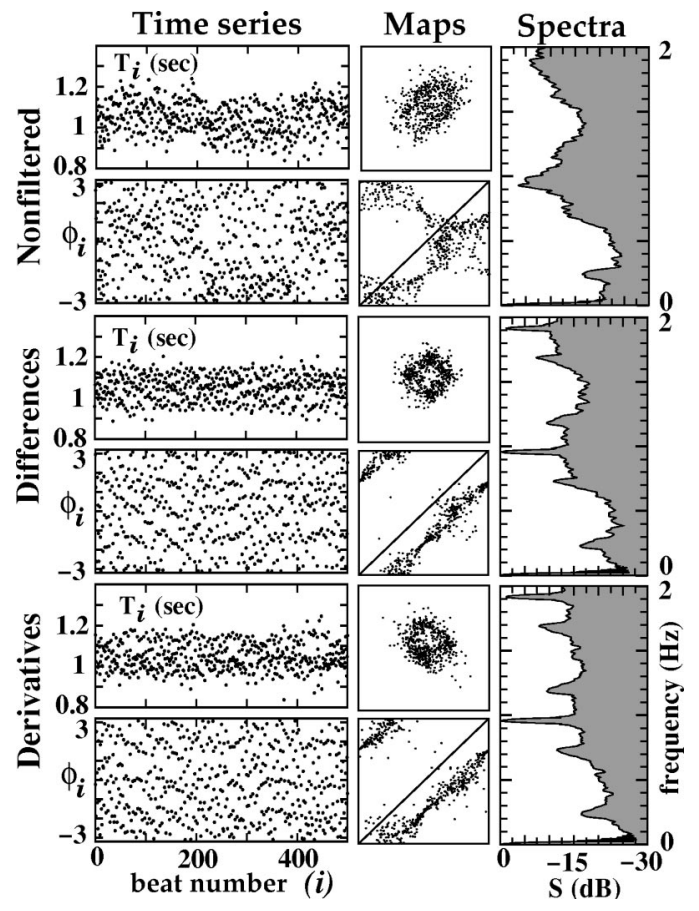

FIG. 4. This figure illustrates the successive stages of $R-R$ data processing and compares the two filtering techniques for a healthy subject at rest. Details are discussed in the text.

region. A typical feature of these maps is the existence of relatively sharp cusps pointing away from the diagonal. The origin of this structure can be explained phenomenologically using the basic property of white noise, namely, the nondifferentiability of its time series. That means that differences between the subsequent points change from negative to positive very quickly. The probability of finding two successive local maxima (or minima) being separated by only one or two points is much larger than the probability of finding a wider separation. An illustration of how the differences technique works in this case is given in Fig. 3(c). In the upper plot the original dataset is shown together with the local average. After subtracting the latter one obtains a time series oscillating around zero with the current period varying randomly between 2 or 3 . In terms of angles maps this means random (and rapid) jumping between the return functions shown in Figs. 3(a) and 3(b). Of course, due to total randomness of the data, the whole map appears to be very noisy. The performance of derivatives technique can be explained in a similar way. Thus, if the shape of the angles map is similar to that presented in Fig. 3, we will consider these data as purely noisy.

\section{STAGES OF DATA PROCESSING}

The stages in the processing of the measured $R-R$ intervals are illustrated in Fig. 4.

An original sequence of $R-R$ intervals is plotted (Fig. 4, first row, first column). As usual, there is a slow variation of the average value, attributable to processes of very low frequency (less than $0.1 \mathrm{~Hz}$ ) which, over small observation times, can often be treated as nonstationarity. On the top right is shown the Fourier power spectrum of the original HRV data [6,7]. The largest peak corresponds to the main heart rhythm $f_{\text {ahr }}$. Both this and the others of smaller amplitude are rather broad, presumably due to the nonstationarity. Correspondingly, the map of successive $R-R$ intervals, being just the return times map (Fig. 4, first row, second column), usually has no distinct structure.

The angles $\phi_{i}$ are extracted from the return times map (Fig. 4, second row, first column), having placed the origin at the center of mass. A typical map for angles is as shown in Fig. 4 (second row, second column), where the points fall close to the diagonal near coordinate positions $(\pi / 4 ; \pi / 4)$ and $(-3 \pi / 4 ;-3 \pi / 4)$ due to the predominance of very lowfrequency oscillations influencing the heart rate [compare with Fig. 6(a) of Part I].

In order to concentrate on interactions of the main rhythm with respiration and the process with frequency $\sim 0.1 \mathrm{~Hz}$, we need to reduce the effect of very low frequencies and subject the original sequence of $R-R$ intervals to filtration. The results of applying two filters described in Sec. III A usually differ slightly, and so in practice we compute the power spectrum for the each of the filtered data sets $x_{\text {der }}$ or $x_{\text {diff }}$ in order to control the effect of filtering. In Fig. 4, rows three and four show the results of filtering by means of differences, while rows five and six illustrate the workability of derivatives technique. The corresponding power spectra are shown at the end of each pair of rows. To compute the Fourier spectrum from filtered $R-R$ intervals we just add to $x_{\text {der }}$ or $x_{\text {diff }}$ the average value of unfiltered $R$ - $R$ intervals, and then proceed in the same way as for original, nonfiltered data [6]. The methods of differences and derivatives both remove the trend from the data, leading to return times maps (rows three and five) of similar appearance. However, the noisy background of the power spectrum seems on average to be more uniform after filtration by derivatives, than by differences, although the use of derivatives leads to a more significant decrease of the lower-frequency range (around $0.1 \mathrm{~Hz}$ and less). In both cases, two dominating frequencies and their combinations are clearly seen after filtration, but the ratios of their amplitudes appear to be slightly different. Note, that the derivatives technique is dangerous for data where the respiration frequency is less than a quarter of average heart rate. For such data we would recommend the use of differences as being the safer method.

The angles $\phi_{i}$ are extracted from the maps of filtered return times, and the map for angles (1) from Part I is plotted (Fig. 4, rows four and six). For the case considered, both angles maps seem to lie in the vicinity of the same curve being the return function of Eq. (11) for in Part I $\xi=\frac{1}{4}$ (compare with Fig. 4). So both methods of filtration allow the structure of angles map in this example to be revealed more or less equally. Both of the "filtered" angles maps represent smeared continuous curves, and are definitely not formed by isolated clouds of points, thus testifying to the absence of synchronization between heart rate and the most dominant of the other processes (which in the present case seems to be respiration). The rotation number $\xi_{01}$ is estimated from Eq. (21) of Part I to be $\langle\xi\rangle=0.246 \ldots$ for differences and $\langle\xi\rangle=0.264 \ldots$ for derivatives. 

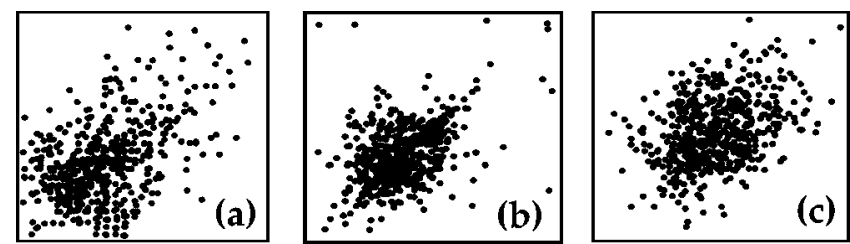

FIG. 5. Map of radii of return times for the subject illustrated by Fig. 12 for (a) nonfiltered $R$ - $R$ intervals, (b) $R$ - $R$ intervals filtered by differences, (c) $R-R$ intervals filtered by derivatives.

For further analysis of real data we select whichever filter leads to the more pronounced structure in the map for angles. Of course, neither of the filtration techniques described is perfect, and other techniques could be used instead to obtain similar or perhaps even better results.

Along with angles, the instantaneous radii were also extracted from the human $R-R$ intervals at each stage of processing. In Fig. 5 the maps for radii $r_{i}$ are shown for the same human data as in Fig. 4: for nonfiltered $R-R$ intervals (a), for those filtered by differences (b) and by derivatives (c). Unlike the angles $\phi_{i}$, the radii $r_{i}$ usually behaved in a rather disordered way, thus smearing the map of $R-R$ intervals significantly. This remained true regardless of whether raw or filtered HRV data were used.

As shown in Part I, the map for angles of return times allows one to make a judgement about synchronization (or its absence) between the main rhythm and the other process with smaller amplitude, interaction with which is dominant. In the CVS the role of this second rhythm is usually played by respiration. The third rhythm often present in human HRV data has a basic frequency $f_{2}$ that is close to $0.1 \mathrm{~Hz}$ and an amplitude comparable with or lower than that due to respiration. In order to obtain information about interaction between respiration and the latter process, if it manifests itself in the power spectrum, we may finally proceed as suggested in Sec. III C of Part I, namely: extract the local maxima from the sequences of $R-R$ intervals; filter the set of maxima by one or another technique; and then plot their map.

\section{TESTING FILTERING ON SURROGATE DATA}

Now, let us test filtering more thoroughly with the help of surrogate data [9]. We are interested in the application of our method to data possessing the same Fourier power spectrum as real $R-R$ intervals, but which is otherwise random. We obtained a set of surrogates for the dataset illustrated in Fig. 4 using the program surrogates from the TISEAN complex developed by the authors of this method [10], and then subjected it to all the same stages of processing. The results are presented in Fig. 6. Fourier spectra of either original or filtered surrogate data possess peaks at the same frequencies as the spectra of the reference data. They are of similar amplitude (compare the spectra in Figs. 4 and 6), although not exactly the same, possibly due to the method of computing the spectrum used here [7], which is not the fast Fourier transform used in [10].

The angles maps look similar to those obtained for the purely random data of Figs. 3(a) and 3(b), and obviously

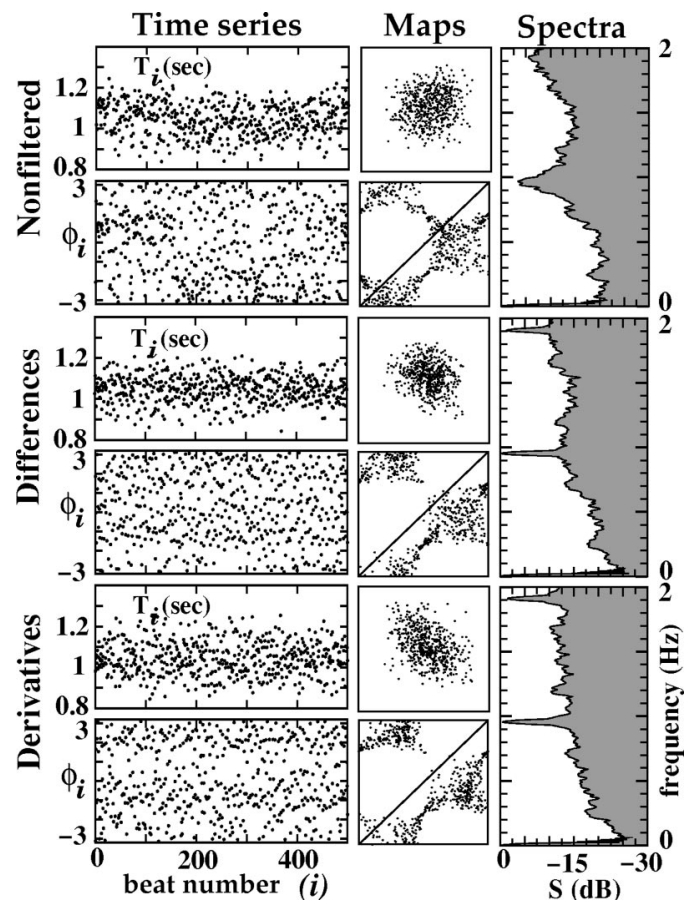

FIG. 6. This figure illustrates the successive stages of processing of surrogate data for the dataset illustrated in Fig. 4 and compares the two filtering techniques. Details are discussed in the text.

differ markedly from the corresponding maps derived from the reference data in Fig. 4.

\section{ANGLES OF RETURN TIMES MAP AND PHASE DIFFERENCE FOR HRV DATA}

In Part I an explicit correspondence between the conventional phase difference and the angles of return times map was derived analytically and, what is of particular importance for us here, confirmed for a simulated nonstationary process with floating eigenfrequency of oscillations. Here we attempt to establish the same correspondence for real biological data. In order to evoke a regime of effective phase synchronization with the same rotation number $\xi=1 / 3$ as in the numerical simulation illustrated in Fig. 7 of Part I, we asked a healthy volunteer to breath at a frequency of $0.5 \mathrm{~Hz}$, and measured both the ECG and respiration signals simultaneously. The Fourier power spectrum of the HRV for this subject is given in Fig. 7, where the two main processes exhibit themselves through the presence of the peaks at $f_{\text {ahr }}$, $f_{\text {resp }}$, and their combinations $2 f_{\text {resp }}=f_{\text {ahr }}-f_{\text {resp }}$. Thus, we

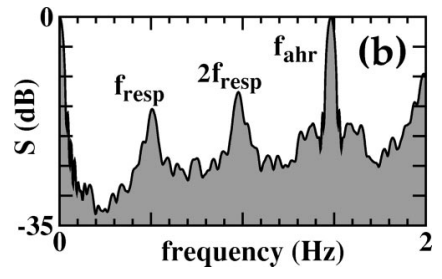

FIG. 7. Fourier power spectrum of a datafile of a subject undergoing paced respiration at frequency $0.5 \mathrm{~Hz}$. Two rhythmic processes dominate: the main cardiac rhythm and respiration. 


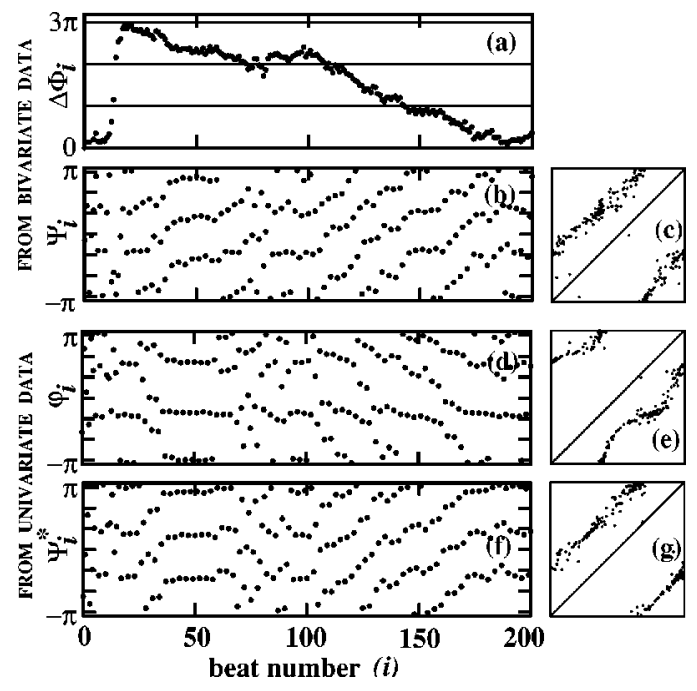

FIG. 8. Comparison of different methods used for the detection of phase synchronization in the human cardiovascular system. The first two rows of plots were derived from bivariate data (ECG and respiration): (a) the conventional phase difference $\Delta \Phi_{i}$ between respiration and ECG; (b) relative phase $\Psi_{i}$; (c) map of relative phase $\Psi_{i+1}$ vs $\Psi_{i}$. The third and fourth rows were obtained from univariate data: (d) angles of map of $R-R$ intervals with removed floating average value; (e) map of angles; (f) angles transformed by means of (8) of Ref. [1]; (g) map of transformed angles. Note the striking similarity between plots (b) and (f), and (c) and (g), respectively.

can suppose that if any synchronization between heart rate and respiration takes place it has the order $1 / 3$. We now proceed to analyze these data in exactly the same way as described in Sec. III A of Part I.

First, we undertake a conventional type of synchronization analysis, computing from the bivariate data the phase difference $\Delta \Phi_{i}=\phi_{\text {ahr }}\left(t_{i}\right)-3 \phi_{\text {resp }}\left(t_{i}\right)$ [Fig. 8(a)]. The moments $t_{i}$ correspond to the appearance of $R$ peaks in the ECG when its phase changes by $2 \pi$, and the phase of respiration signal is taken as the phase angle of a phase portrait reconstructed by a suitable delay embedding from the respiration signal. We then construct the relative phase $\Psi_{i}$ and its map [Figs. 8(b) and 8(c), respectively]. In Figs. 8(a) and 8(b) we can notice several horizontal segments testifying to the occurrence of phase locking, and intervals where two phases slide against each other, i.e., are not locked.

Secondly, we perform the new type of synchronization analysis proposed in Part I, restricting ourselves to univariate data only. We choose for the latter the $R-R$ intervals extracted from the ECG. We filter them by the derivatives method, extract angles $\phi_{i}$ and plot their map [Figs. 8(d) and 8(e), respectively]. Finally, we transform $\phi_{i}$ using Eq. (8) of Part I to "reconstruct" the relative phase $\Psi_{i}^{*}$ whose temporal dependence and map are shown in Figs. 8(f) and 8(g), respectively [11].

The striking similarity between the plots in Figs. 8(b) and $8(\mathrm{f})$, and $8(\mathrm{c})$ and $8(\mathrm{~g})$ provides a convincing demonstration that the angles of a return times map are able to provide the same information as conventional phase difference and relative phase. In other words, we have indeed been able to

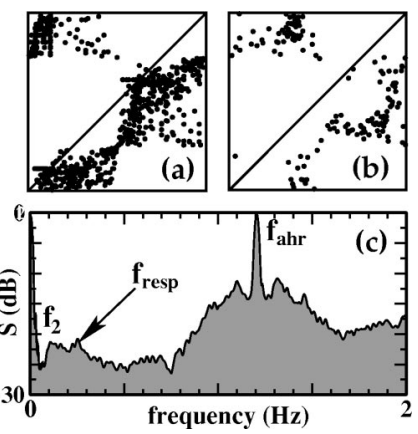

FIG. 9. Example of a datafile where three time scales are important, but no pair of them is synchronous. (a) Map of angles of $R-R$ intervals. (b) Map of angles extracted from the map of all local maxima of $R-R$ intervals. (c) Fourier power spectrum.

extract essentially the same information about synchronization from the univariate time series as we obtained from two time series analyzed in the conventional way.

\section{SOME EXAMPLES OF EXPERIMENTAL ANGLE MAPS}

In this section we present and discuss two examples of different phase and amplitude relationships between the three processes interacting within the CVSs of particular subjects.

The first example is illustrated by Fig. 9. The $R-R$ intervals are subjected to filtering by differences here, and the corresponding angles map in given in Fig. 9(a). The Fourier spectrum [Fig. 9(c)] reveals three distinct frequency components $f_{\text {ahr }}, f_{\text {resp }}$, and $f_{2}$, and the angles map (a) is not close to any one-dimensional curve. Neither does it contain isolated clouds of points, so one can be confident that there is no phase locking between the main heart rhythm and respiration. Since there are three rhythms involved in the interaction, there are two independent rotation numbers, namely, $\xi_{01}$ for the interaction between heart beat and respiration, and $\xi_{12}$ for the interaction between respiration and the process with frequency $f_{2}$. Formula (21) of Part I, which is suitable for only two interacting processes, is not expected to provide a reliable estimate for any of the true "partial" rotation numbers. However, the average rotation number $\langle\xi\rangle=0.2060 \ldots$ seems to lie close to the ratio of the heart rate and respiration frequency.

Now, consider the interaction between respiration and the process with $f_{2}$. Extract local maxima from the original sequence of $R-R$ intervals, filter them by derivatives; extract angles, and create their map [Fig. 9(b)]. A one-dimensional structure is quite evident here, although it cannot be described by Eq. (11) in Part I (cf. the plots in Fig. 3 of Part I). The probable reason is that the amplitude of the process with $f_{2}$ is not much less than that of respiration, but is comparable with it. Thus approximation of Eq. (11) in Part I is no longer valid, and we have no right to apply formula (21) of Part I to estimate the rotation number. The observed map contains no isolated groups of points and can be taken as evidence for the absence of phase locking between respiration and $f_{2}$. Thus, in the example considered no two of the three processes involved are synchronized with each other. 


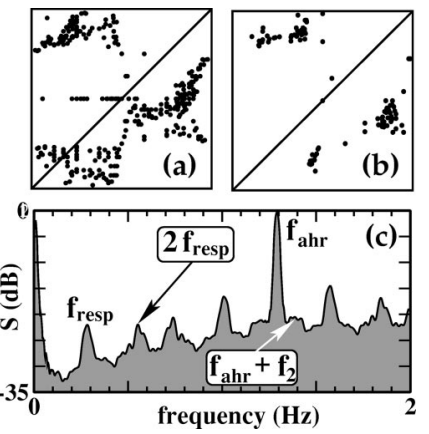

FIG. 10. Example of a datafile where three time scales are important. Respiration is not synchronous with heart rate, but is synchronous with the rhythm whose $f_{2}=0.1 \mathrm{~Hz}$. (a) Map of angles of $R-R$ intervals. Note: the interaction between heart rate and respiration is nonlinear, and so the map is not captured by Eqs. (11) or (19) in Part I. (b) Map of angles extracted from the map of all local maxima of $R-R$ intervals (note the distinct clouds of points). (c) Fourier power spectrum [note the distinct second harmonic of respiration frequency $2 f_{\text {resp }}$ and the combination $\left.\left(f_{\text {ahr }}-2 f_{\text {resp }}\right)\right]$.

The second example is illustrated by Fig. 10. The power spectrum [Fig. 10(c)] contains distinguishable components of the main rhythm $f_{\text {ahr }}$ and respiration $f_{\text {resp }}$, and much less pronounced combination frequencies $f_{\text {ahr }} \pm f_{2}$. The first angles map [Fig. 10(a)] reveals a structure that is close to being one dimensional but which is not, however, captured by model (11) in Part I (compare with plots in Fig. 3). The reason is that, as in case of Fig. 10(b), interaction between the main process and respiration cannot be treated as weak; the latter conclusion is supported by the presence at relatively large amplitude of second harmonics of the respiration frequency $2 f_{\text {resp }}$ and also the combination frequency ( $f_{\text {ahr }}$ $\left.-2 f_{\text {resp }}\right)$. In this case too we cannot estimate the rotation number $\xi_{01}$ by means of Eq. (21) of Part I. However, in spite of the rather strong interaction, no synchronization between the basic process and respiration can be detected, since the angles map is close to a continuous curve. We now eliminate the main rhythm by selecting local maxima of $R-R$ intervals, filter them by derivatives, and plot the corresponding map for angles [Fig. 10(b)]. It clearly contains several isolated clouds of points, whose exact number will be discussed below: they constitute evidence of phase locking between the processes considered. The average rotation number $\xi_{12}$ can be estimated as $\langle\xi\rangle=0.3936 \ldots$ that is close to $2 / 5$.

Since the numerator of the rotation number, if synchronization exists, seems to be $n=2$, let us apply the technique used in Ref. [12] to detect phase locking. Namely, unwrap the angles allowing them to increase monotonically, and then wrap them into an interval $[-2 \pi ; 2 \pi]$ that is twice as large as $[-\pi ; \pi]$ [Fig. 11(a)]. Now, compute the probability density for this dependence [Fig. 11(b)]. We find that it posseses 5 distinct peaks. This allows us to infer the existence of $2 / 5$ synchronization between respiration and the process with $f_{2}$, at least in the statistical sense [12].

\section{MODELING ANGLES OF $R$ - $R$ INTERVALS}

Let us apply the theoretical map (19) of Part I to simulate the observed angles maps. For simplicity we set all phase

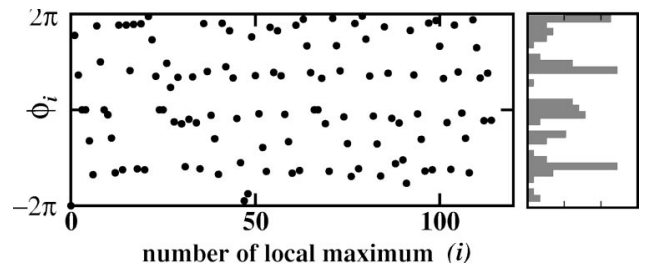

FIG. 11. (a) Angles of the "secondary" return times map for a subject illustrated by Fig. 10, extended to the interval $[-2 \pi ; 2 \pi]$. The map of these angles is given in Fig. 10(b). (b) Probability distribution of these angles, showing five peaks.

shifts $\phi_{j}^{0}$ to zero. First, we simulate the map of Fig. 8(e) by setting frequencies $\omega_{0}=1, \Omega_{1}=1 / 3, \Omega_{2}=0.1$; amplitudes $A_{1}=0.1, A_{2}=0.01$; the intensity of Gaussian white noise modulating the value of $\Omega_{1}$ as $D=0.00002$, the noise added to the right-hand part of model map $D=0.06$, and also some "measurement noise" added to the solution with intensity $D=0.05$. Since the number of points in Fig. 8 is about 200, for a good comparison the same number of points of the map (19) in Part I with the given parameter values are presented in Fig. 12(a). The two phase portraits are evidently very similar.

Secondly, we simulate the case of Fig. 10(b) by setting: frequencies $\omega_{0}=1, \Omega_{1}=0.4, \Omega_{2}=0.112 \ldots$ (a long random sequence of numbers from 1 to 9); amplitudes $A_{1}=0.2, A_{2}$ $=0.05$; and the intensity of Gaussian white noise added to the equation $D=0.001$. 100 points of the resulting phase portrait are given in Fig. 12(b): the result looks remarkably similar to that in Fig. 10(b). Note, that the rotation number $\xi_{12}$ here is set to exactly $2 / 5$, and the tendency to merge for the two clouds of points furthest to the right is clearly seen. This latter example serves as an argument supporting our inference of $2 / 5$ phase locking between respiration and $f_{2}$.

Thus, the derived general map (18) from Part I [and its particular case (19)] allows the dynamics of real cardiovascular signals to be modeled, at least in those cases where the main process interacts sufficiently weakly with the others.

\section{DISCUSSION}

The results presented in some sense contradict to the earlier conclusion [13] that no distinct structure arises in the angles maps of human $R-R$ intervals in the case of spontaneous breathing, and can appear only for paced respiration at
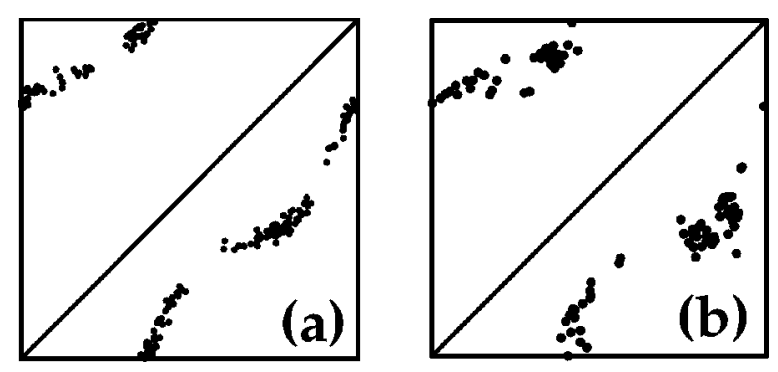

FIG. 12. Two examples of modeling the angles maps by means of Eq. (19) of Part I. Model of map in (a) Fig. 8(e), and (b) Fig. $10(\mathrm{~b})$. 
frequencies close to $0.1 \mathrm{~Hz}$. We have demonstrated above that, although structure cannot be seen in the raw results, filtration of the data to remove the floating average value enables distinct structure to be observed for most healthy subjects. Thus, the deterministic structure revealed in angles map of filtered $R$ - $R$ intervals is the evidence of deterministic interaction between heart rate and, most probably, respiration and the oscillatory process at $0.1 \mathrm{~Hz}$.

\section{SUMMARY AND CONCLUSIONS}

Based on the results presented above, we arrive at the following conclusions:

(1) In experimental heart-rate-variability data of healthy humans, the instantaneous radii $r_{i}$ are rather disordered, whereas the angles $\phi_{i}$ of return times reveal much determinism in most of the cases considered.

(2) The majority of the HRV data analyzed were successfully modeled by the formulas (11) and (19) of Part I which was derived for the case of weak interaction. That means that interaction of the processes involved can be considered weak.

(3) There are some data that contain distinct structure that is not captured by our models, thus revealing the existence of stronger interactions in some cases.

(4) The technique presented allows one to study synchro- nization between at least three processes interacting within the cardiovascular system.

The cardiovascular system is a particularly striking example of a system within which several oscillatory processes interact, mutually influencing each other. With the exception of respiration and the main cardiac rhythm, there is no possibility of separating the signals from the individual processes in order to compare them and assess their synchronization, or the lack of it, using conventional techniques. We have suggested and justified theoretically a tool $[1,2]$ to study interacting rhythms in the cardiovascular system using only heart rate variability data. We expect that the same approach will be equally applicable to the other kinds of biomedical signals with less or comparably complex structure. We hope that the proposed approach may prove to have potential for future applications and the development of new criteria for use in medical diagnostics.

\section{ACKNOWLEDGMENTS}

We are much indebted to Dr. Alexander Neiman for valuable discussions and for his constructive comments on a draft version of the manuscript. The work was supported by the Engineering and Physical Sciences Research Council (UK), the Leverhulme Trust, the Medical Research Council (UK), and the U.S. Civilian Research Development Foundation (Award No. REC 006).
[1] N. B. Janson, A. G. Balanov, V. S. Anishchenko, and P. V. E. McClintock, Phys. Rev. Lett. 86, 1749 (2001).

[2] N. B. Janson, A. G. Balanov, V. S. Anishchenko, and P. V. E. McClintock, preceding paper, Phys. Rev. E 65, 036211 (2002).

[3] S. Akselrod, D. Gordon, J. B. Madwed, N. C. Snidman, C. S. Shannon, and R. J. Cohen, Am. J. Physiol. 249, H867 (1985); B. Pomeranz et al., ibid. 248, H151 (1985).

[4] B. W. Hyndman, R. I. Kitney, and McA. Sayers, Nature (London) 233, 339 (1971).

[5] A. Stefanovska and M. Bračič, Contemp. Phys. 40, 31 (1999).

[6] The Fourier spectrum of a sequence of interspike intervals was computed as suggested in Ref. [7]. Namely, the signal to be processed was presented as a sum of $\delta$ spikes placed at the time moments when $R$ peaks occured in the electrocardiogramme. The Fourier transform was then applied to the resultant signal using its mathematical definition via an integral. This procedure allows one to pass from dimensionless frequen- cies to real frequencies in hertz and to consider the full spectrum, unrestricted by the usual 0.5 /beat defined by the Nyquist theorem for discrete data.

[7] R. W. DeBoer, J. M. Karemaker, and J. Strackee, IEEE Trans. Biomed. Eng. 31, 384 (1984).

[8] A. Stefanovska and M. Hožič, Prog. Theor. Phys. Suppl. 139, 270 (2000).

[9] T. Schreiber and A. Schmitz, Physica A 142, 346 (2000).

[10] R. Hegger, H. Kantz, and T. Schreiber, Chaos 9, 413 (1999).

[11] As noted in Part I [2], $\Psi_{i}^{*}$ defines the relative phase up to some constant. While plotting Fig. 8(f) we added to $\Psi_{i}^{*}$ a shift 0.3 chosen by trial in order to gain maximal similarity with Fig. 8(b).

[12] C. Schäfer, M. G. Rosenblum, J. Kurths, and H.-H. Abel, Nature (London) 392, 239 (1998).

[13] K. Suder, F. R. Drepper, M. Schiek, and H.-H. Abel, Am. J. Physiol.-Heart. C 44 (3), H1092 (1998). 